\title{
Interphalangeal Joint 4 of the Foot
}

National Cancer Institute

\section{Source}

National Cancer Institute. Interphalangeal Joint 4 of the Foot. NCI Thesaurus. Code

C114203.

A ginglymoid (hinge) synovial joint between the phalanges of the fourth digit of the foot. 